dusts have a role in the aetiology of cryptogenic fibrosing alveolitis. Several metal dusts have been implicated as causes of pulmonary fibrosis, ${ }^{14}$ but whether in our patients metal and wood dusts were true fibrotic agents or markers of exposure to other fibrogens is not clear. For example, workers exposed to metal dust are often also exposed to grinding or lubricating materials, and joiners exposed to wood dust may also work with insulation or roofing materials containing asbestos.. In relation to exposure to cattle, cryptogenic fibrosing alveolitis in a dairy worker has previously been attributed to occupational exposure to hydrogen peroxide. ${ }^{15}$ Clearly, the extent of confounding by exposure to other agents needs to be explored, but the necessary detail is not available in the present study. Our finding that patients with cryptogenic fibrosing alveolitis had had a higher exposure to domestic wood fires adds support to a suggestion that wood smoke may be a cause of the disease. ${ }^{16}$ Cigarette smoke, however, had no appreciable effect in the present study despite an apparent protective effect against lung fibrosis due to extrinsic allergic alveolitis or sarcoidosis. ${ }^{17} 18$

The relevance of the increased reporting of allergic symptoms by our patients is not clear as shortness of breath on exposure to allergens may be a non-specific symptom and recall bias may also have contributed. Further investigation of this is therefore indicated. Future studies should also investigate whether disease associated with inhalation of dust differs in terms of histological findings or clinical progress from the classic rapidly progressing form of the disease as cryptogenic fibrosing alveolitis might be a stereotyped end organ response to many different aetiological factors. The present evidence associating the disease with potentially avoidable exposure to environmental agents, however, raises the possibility that it may at least to some extent be preventable.
We thank Dr Jim Pearson for his advice on computing and statistics, Mrs Mary Stevenson for entering and verifying the data, and Mrs Bernadette Maughan and others of the family practitioner committee for selecting the controls.

Copies of the questionnaire are available on request.

1 Crystal RG, Bitterman PB, Rennard SI, Hance AJ, Keogh BA. Interstitial lung diseases of unknown cause. Part I. N Engl f Med 1984;310:154-63.

2 Grant IWB. Cryptogenic fibrosing alveolitis. In: Weatherall DJ, Ledingham JGG, Warrell DA, eds. Oxford textbook of medicine. 2nd ed. Oxford: Oxford University Press: 1987:15.123-5.

3 Stack HR, Choo-Kang YFJ, Heard BE. The prognosis of cryptogenic fibrosing alveolitis. Thorax 1972;27:535-42.

4 Turner-Warwick $M$, Burrows B, Johnson A. Cryptogenic fibrosing alveolitis: clinical features and their influence on survival. Thorax 1980;35:171-80.

5 Crystal RG, Fulmer JD, Roberts WC, Moss ML, Line BR, Reynolds HY Idiopathic pulmonary fibrosis: clinical, histologic, radiographic, physiologic, scintographic, cytologic and biochemical aspects. Ann Intern Med 1976;85:769-88.

6 Chetty A, Bhuvan UN, Mitra DK, Roy S, Deorati A. Cryptogenic fibrosing alveolitis in children. Ann Allergy 1987;58:336-40.

7 Johnston IDA, Britton JR, Kinnear WJM, Logan RFA. Rising mortality from cryptogenic fibrosing alveolitis. Br Med f 1990;301:1017-21.

8 SPSS Inc. Statistical package for the social sciences-SPSS-X: user's guide. $2 \mathrm{nd}$ ed. New York: McGraw Hill, 1986.

9 Office of Population Censuses and Surveys. Classification of occupations 1980. London: HMSO, 1980.

10 Anonymous. EGRET reference manual. First draft. Seattle: Statistics and Epidemiology Research Corporation and Cytel Software Corporation, 1990 Robinson BWS, Rose AH. Pulmonary gamma-interferon production in patients with fibrosing alveolitis. Thorax 1990;45:105-8

12 Bleasdale C, Johnston IDA, Kara S, Woodcock AA, Evans CC, Hind CRK. Management of patients with cryptogenic fibrosing alveolitis in the 1980s: experience in three regions. Thorax 1989;44:346P.

13 Liebow AA, Steer A, Billingsley JG. Desquamative interstitial pneumonia. Am f Med 1965;39:369-404.

14 Morgan WKC, Seaton A. Occupational lung diseases. London: W B Saunders, 1984.

15 Kaelin RM, Kapanci Y, Tschopp JM. Diffuse interstitial lung disease associated with hydrogen peroxide inhalation in a dairy worker. Am Rev Respir Dis 1988;137:1233-5.

16 Ramage JE, Roggli VL, Bell DY, Piantadosi CA. Interstitial lung disease and domestic wood burning. Am Rev Respir Dis 1988;137:1229-32

17 Depierre A, Dalphin JC, Pernet D, Dubiez A, Faucompre C, Breton JL. Epidemiological study of farmer's lung in five districts of the French Daubs province. Thorax 1988;43:429-35.

18 Valeyre D, Soler P, Clarici C, et al. Smoking and pulmonary sarcoidosis: effect of cigarette smoking on prevalence, clinical manifestations, alveolitis and evolution of the disease. Thorax 1988;43:516-24.

(Accepted 15 August 1990)
University Hospital, Nottingham NG7 2UH Ian Johnston, $\mathrm{MD}$, consultant physician

William Kinnear, MD, senior registrar

Respiratory Medicine Unit, City Hospital, Nottingham NG5 1PB

John Britton, MD, lecturer

Department of Community Medicine and

Epidemiology, Queen's

Medical Centre,

Nottingham NG7 2UH

Richard Logan, FRCPED, senior lecturer

Correspondence to: Dr Johnston.

BrMed f 1990;301:1017-21

\title{
Rising mortality from cryptogenic fibrosing alveolitis
}

\author{
Ian Johnston, John Britton, William Kinnear, Richard Logan
}

Abstract
Objective-To determine the pattern of mortality ascribed to cryptogenic fibrosing alveolitis and to identify factors that might be important in the aetiology of the disease; and to assess the validity of death certification of the disease.

Design - A retrospective examination of mortality ascribed to cryptogenic fibrosing alveolitis in England and Wales between 1979 and 1988 with analysis, by multiple logistic regression, of independent effects of age, sex, region of residence, and social class as indicated by occupation on data for 1979-87; also a retrospective review of hospital records of patients certified as having died of cryptogenic fibrosing alveolitis in Nottingham and of the certified cause of death of patients known to have had the disease.

Main outcome measures - Time trends in mortality nationally; effects on mortality of age, sex, and region of residence; validity of death certification in Nottingham.

Results - The annual number of deaths ascribed to cryptogenic fibrosing alveolitis doubled from 336 in 1979 to 702 in 1988, the increase occurring mainly at ages over 65 . Mortality standardised for age for both sexes likewise increased steadily over the period. Deaths due to cryptogenic fibrosing alveolitis were commoner in men (odds ratio $2 \cdot 24,95 \%$ confidence interval $2 \cdot 11$ to 2.33 ) and increased substantially with age, being $7.84(7.24$ to 8.49$)$ times higher in subjects aged $\geqslant 75$ than those aged 45-64. Odds ratios of death due to cryptogenic fibrosing alveolitis adjusted for age and sex were increased in the traditionally industrialised central areas of England and Wales $(p<0.02$, maximum odds ratio between regions 1.25 ), but no significant increase in odds of death was found for manual occupations. Of 23 people whose deaths were registered in Nottingham as having been due to cryptogenic fibrosing alveolitis, 19 were ascertained from clinical records to have had the disease. Only 17 of 45 patients known to have had cryptogenic fibrosing alveolitis in life were recorded as having died from the disease.

Conclusions-The diagnostic accuracy of death certification of cryptogenic fibrosing alveolitis is high, but the number of deaths recorded as being due to the disease may underestimate the number of patients dying with the disease by up to half. Mortality due to the disease is increasing, and the male predominance and regional differences in mortality suggest that environmental factors are important in its aetiology.

\section{Introduction}

The aetiology of cryptogenic (idiopathic) fibrosing alveolitis is unknown, and the mean life expectancy of a patient after presentation with the disease is only four 
to five years. ${ }^{1-3}$ The disease has been investigated extensively at the biochemical and cellular levels ${ }^{14}$ but has attracted remarkably little epidemiological interest; indeed, until fairly recently it was not classified as a distinct entity in the ICD..$^{5}$ The introduction in 1979 of a specific ICD code (516.3) for the disease provided the opportunity to examine trends in mortality, and inspection of the data showed that in England and Wales the number of deaths attributed to cryptogenic fibrosing alveolitis doubled between 1979 and 1988. We analysed the national data on mortality to describe the pattern of mortality from cryptogenic fibrosing alveolitis and to identify possible causal factors. To assess the validity of death certification of cryptogenic fibrosing alveolitis we compared clinical records and death certification for the disease in the Nottingham area.

\section{Methods \\ NATIONAL MORTALITY DATA}

We calculated annual mortality from cryptogenic fibrosing alveolitis specific for age and sex from the routinely published mortality statistics for England and Wales for $1979-87^{6}$ and from provisional figures for 1988 obtained direct from the Office of Population Censuses and Surveys. We used the structure of the population in 1979 to calculate rates standardised for age.

FIG 1-Number of deaths coded as due to cryptogenic fibrosing alveolitis and mortality from the disease $110^{7}$ men and women standardised for age to 1979 , England and Wales, 1979-88

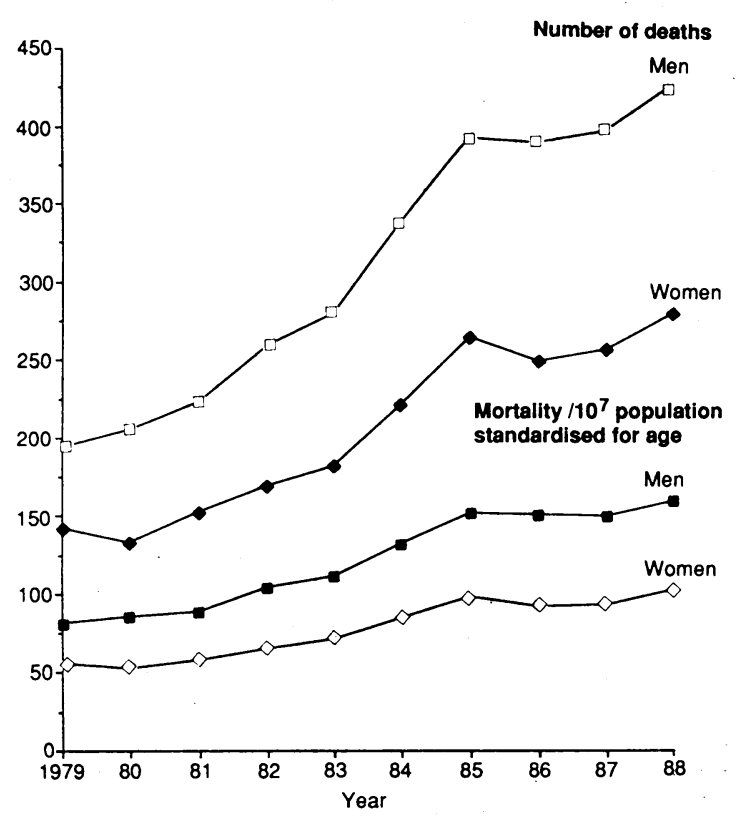

TABLE I-Coefficients obtained by multiple logistic regression, odds ratios, and results of $\chi^{2}$ test for effects of age, sex, and standard region of residence on mortality from cryptogenic fibrosing alveolitis in England and. Wales, 1979-87

\begin{tabular}{|c|c|c|c|c|c|c|}
\hline & $\begin{array}{l}\text { Estimate from } \\
\text { multiple logistic } \\
\text { regression }\end{array}$ & SE & $\begin{array}{l}\text { Odds } \\
\text { ratio }\end{array}$ & $\chi^{2}$ & df & $\mathrm{p}$ Value \\
\hline \multicolumn{7}{|l|}{$\begin{array}{l}\text { Intercept (female sex, age } 0-14 \text {, resident } \\
\text { in north region) }\end{array}$} \\
\hline \multicolumn{7}{|l|}{ Addition for: } \\
\hline Age (years) & & & & 11139 & 4 & $<0.001$ \\
\hline $15-$ & 0.73 & $0 \cdot 30$ & $2 \cdot 1$ & & & \\
\hline $45-$ & $4 \cdot 14$ & 0.28 & $62 \cdot 8$ & & & \\
\hline $65-$ & 5.67 & $0 \cdot 28$ & $290 \cdot 0$ & & & \\
\hline$\geqslant 75$ & $6 \cdot 20$ & 0.28 & $491 \cdot 3$ & & & \\
\hline Male sex & 0.81 & 0.03 & $2 \cdot 24$ & 694 & 1 & $<0.001$ \\
\hline Standard region of residence & & & & $19 \cdot 8$ & 8 & $<0.02$ \\
\hline Yorkshire and Humberside & -0.04 & 0.08 & 0.96 & & & \\
\hline East midlands & $0 \cdot 16$ & 0.08 & $1 \cdot 18$ & & & \\
\hline East Anglia & 0.03 & $0 \cdot 10$ & 1.03 & & & \\
\hline South east & -0.04 & 0.07 & 0.96 & & & \\
\hline South west & -0.07 & 0.08 & 0.94 & & & \\
\hline West midlands & 0.05 & 0.08 & 1.06 & & & \\
\hline North west & 0.08 & 0.07 & 1.08 & & & \\
\hline Wales & 0.06 & 0.09 & 1.06 & & & \\
\hline
\end{tabular}

We also obtained from the Office of Population Censuses and Surveys photocopies of the microfiche frames giving the individual details of all deaths coded as having been due to cryptogenic fibrosing alveolitis in England and Wales from 1979 to 1987. Details available were sex, age and region of residence at death, occupation, and Registrar General's social class. Data were entered by double entry key punching into the mainframe computer system at Nottingham University. Basic editing and cross tabulation were done with the Statistical Package for the Social Sciences-X (SPSS-X). ${ }^{7}$ From the pooled data for 1979-87 mortalities by age, sex, and standard region of residence were compared by multiple logistic regression using the generalised linear interactive modelling program. ${ }^{8}$ Estimates of the population in 1984 (roughly the middle of the study) were used as denominators. ${ }^{6}$ Mortality among men aged under 75 was analysed by social class (non-manual or manual occupation), as well as by age and region of residence, by using the regional estimates of population by social class derived from the 1981 census' $^{\text {; }}$ effects of social class were not analysed in women because social class was available for only a small number of those who died (242).

Few deaths occurred in subjects under age 45, but exclusion of these from the multiple regression analyses made little difference to the relative odds of death in any of the comparisons made, so results are presented for the complete age range. In view of the comparative rarity of the disease under study odds ratios were assumed to approximate true relative risks.

\section{VALIDITY OF DEATH CERTIFICATION}

From computer records of all deaths registered in Nottingham we identified those patients who died from October 1985 to December 1987 for whom the cause of death was recorded as cryptogenic fibrosing alveolitis (ICD code 516.3 ; 25 patients) or postinflammatory pulmonary fibrosis (code 515.9; 24 patients). The hospital case records were then reviewed and categorised according to four criteria: (1) definite cryptogenic fibrosing alveolitis-inspiratory crackles on auscultation of the chest, radiographic shadowing in the lower zone of the lungs, and restrictive lung function with no significant history of relevant occupational or other exposure to known fibrogenic agents; (2) possible cryptogenic fibrosing alveolitisinspiratory crackles on auscultation of the chest and radiographic shadowing in the lower zone of the lungs, but no record of lung function or details of occupational or other exposure; (3) fibrosing alveolitis associated with a defined connective tissue disease; (4) not cryptogenic fibrosing alveolitis (that is, not meeting criteria 1-3).

From a register of patients with cryptogenic fibrosing alveolitis in Nottingham (compiled from clinical records and laboratory records of pulmonary function, Hospital Activity Analysis, and Körner admission data) we identified 48 patients whom we knew had had definite cryptogenic fibrosing alveolitis and had died (though not necessarily from the disease) during 1985-9. Death certificates were obtained for these patients and examined for mention of cryptogenic fibrosing alveolitis or pulmonary fibrosis.

\section{Results}

\section{NATIONAL MORTALITY DATA}

In England and Wales 5135 deaths were coded as being due to cryptogenic fibrosing alveolitis from 1979 to 1988 , of which $3093(60 \%)$ were in men. The annual number of deaths increased substantially from 336 in 1979 to 702 in 1988 (fig 1). Mortality standardised for age likewise increased steadily over the period in both sexes (fig 1). The increase in mortality was particularly 

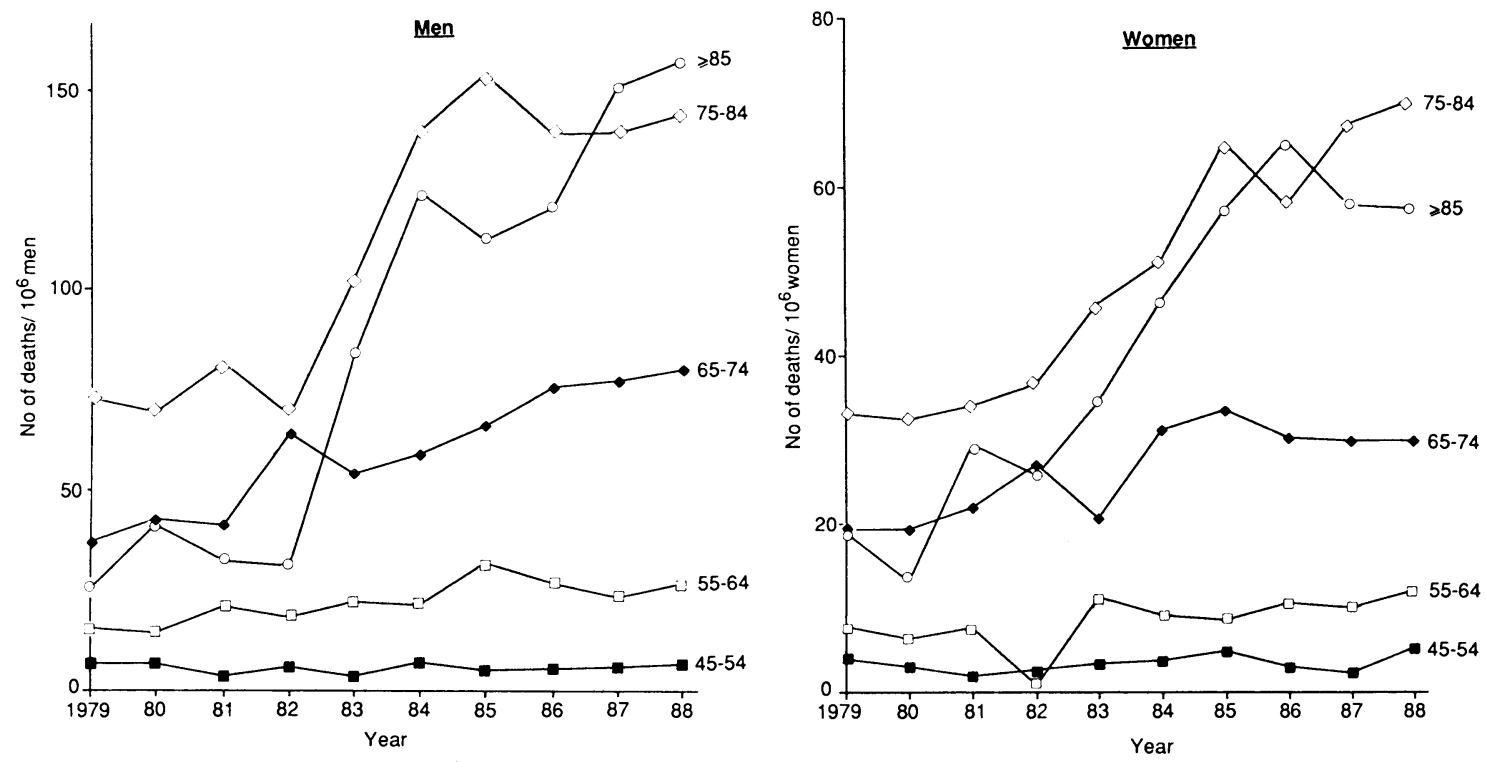

FIG 2-Mortality by age from cryptogenic fibrosing alveolitis/10' men and women, England and Wales, 1979-88

FIG 3-Ranking of standard regions of England and Wales fibrosing alveolitis ( 1 indicates highest mortality) by mortality from cryptogenic

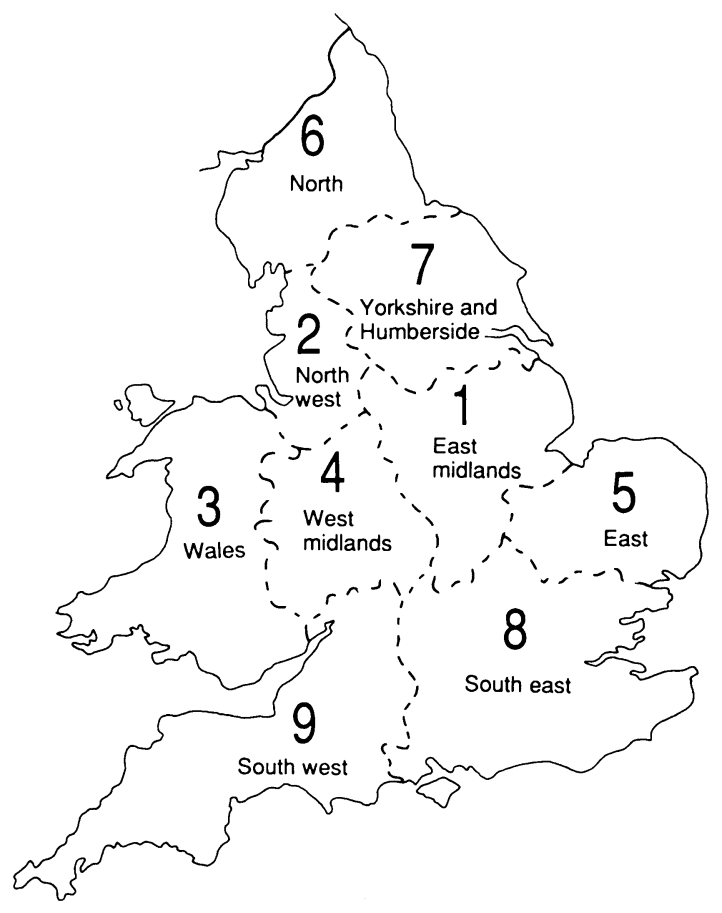

TABLE II-Validity of certification of deaths as being due to cryptogenic fibrosing alveolitis or postinflammatory pulmonary fibrosis in Nottingham, 1985-7

\begin{tabular}{lcc}
\hline & \multicolumn{2}{c}{ Death coded as due to: } \\
\cline { 2 - 3 } & $\begin{array}{c}\text { Cryptogenic fibrosing } \\
\text { alveolitis }\end{array}$ & $\begin{array}{c}\text { Post-inflammatory } \\
\text { pulmonary fibrosis }\end{array}$ \\
\hline Total No of deaths & 25 & 24 \\
No for which hospital records available & 23 & 20 \\
Cause of death as classified on review of hospital records: & 14 & 6 \\
Definite cryptogenic fibrosing alveolitis & 5 & 3 \\
Possible cryptogenic fibrosing alveolitis & 1 & 2 \\
Fibrosing alveolitis with connective tissue disease & 3 & 9 \\
Not cryptogenic fibrosing alveolitis & & \\
\hline
\end{tabular}

TABLE III-Diseases mentioned in death certificates of patients in Nottingham known to have had cryptogenic fibrosing alveolitis and to have died during 1985-9

\begin{tabular}{lc}
\hline & No \\
\hline No known to have had cryptogenic fibrosing alveolitis & 48 \\
No for whom death certificates available & 45 \\
Diseases mentioned in death certificate: & 17 \\
Cryptogenic fibrosing alveolitis or fibrosing alveolitis given as underlying cause of death & 8 \\
Cryptogenic fibrosing alveolitis or fibrosing alveolitis mentioned but not as underlying cause of & 3 \\
$\quad$ death & 17 \\
Pulmonary fibrosis given as underlying cause of death & 3 \\
\hline
\end{tabular}

evident in the older age groups in both sexes (fig 2).

Complete data on age, sex, and region of residence were available for 4411 of the 4433 people who died of the disease from 1979 to 1987 . The odds of death were higher in men by a ratio of $2 \cdot 24$ (95\% confidence interval $2 \cdot 11$ to $2 \cdot 38$ ) and increased progressively with age; mortality in those aged $\geqslant 75$ was roughly double that in those aged 65-74 and eight times that in those aged 45-54 (table I). The regional number of deaths varied between 185 in East Anglia and 1442 in the south east; after adjustment for age and sex there were small but significant residual differences in mortality between the regions, the odds ratios between the highest and lowest being $1 \cdot 25$ (table I, fig 3 ).

Data on manual or non-manual occupation were available for 1578 men and 242 women aged 15-74; social class codes were not provided for people who died aged over 74. In men, after allowance for age, social class had virtually no independent effect on mortality (odds ratio 1.02 for manual relative to nonmanual occupation) and a negligible effect on odds ratios for the region of residence.

\section{VALIDITY OF DEATH CERTIFICATION}

Case records were available for 23 of the 25 patients coded as having died of cryptogenic fibrosing alveolitis whose deaths had been registered in Nottingham. According to the criteria that we used, 19 patients had had definite or possible cryptogenic fibrosing alveolitis and only three clearly had not had the disease (table II). Nine of those coded as having died of post-inflammatory pulmonary fibrosis had had definite or possible cryptogenic fibrosing alveolitis.

Death certificates were traced for 45 of the 48 patients with cryptogenic fibrosing alveolitis in Nottingham whom we knew from clinical records had died during 1985-9. The disease was mentioned in only 25 certificates and given as the underlying cause of death in 17 (table III).

\section{Discussion}

Our study shows that from 1979 to 1988 the number of deaths reported to be due to cryptogenic fibrosing alveolitis increased substantially and that the disease is an important contributor to the overall mortality from respiratory diseases. These findings raise the questions of whether this increase in mortality is a true increase and what the underlying cause of the disease might be.

To assess the validity of the national figures we examined the diagnostic accuracy of death certification 
in the Nottingham area. We found that most patients coded as having died from cryptogenic fibrosing alveolitis had indeed had the disease, defined by criteria similar to those used by others. ${ }^{2}$ A substantial proportion of those coded as having had postinflammatory pulmonary fibrosis, however, had also had cryptogenic fibrosing alveolitis by these criteria, so that the total number of deaths registered as being due to the disease slightly underestimated the true number. Similar misclassification of coding has also been shown for admissions to hospital for the disease..$^{10}$ For less than half of a group of patients known to have had the disease, however, was the cause of death recorded as cryptogenic fibrosing alveolitis or pulmonary fibrosis. Though some patients will have died from unrelated causes, our evidence suggests that the number of deaths reported underestimates the actual number of deaths of people with cryptogenic fibrosing alveolitis (but not necessarily deaths due to the disease) by about half. Assuming that the data for Nottingham are broadly representative, we therefore estimate that in England and Wales some 1300 people with cryptogenic fibrosing alveolitis die each year. Thus the national mortality data probably have high diagnostic validity but there remains considerable scope for artefactual changes in the number of deaths reported to be due to cryptogenic fibrosing alveolitis as this will vary with the proportion of patients in whom the disease is considered to be the actual cause of death.

How much of this increased mortality can be attributed to changes in coding and rules on coding? The introduction of the new code for cryptogenic fibrosing alveolitis in 1979 cannot itself have accounted for the increase as the mortality increased steadily through the subsequent decade. In 1984 the Office of Population Censuses and Surveys changed the rules on coding according to rule 3 of the World Health Organisation, so that if the cause of death was recorded as, for example, unspecified pneumonia or bronchopneumonia in part 1 of the death certificate the disease mentioned in part 2 became the underlying cause of death. Duplicate coding of all deaths ascribed to cryptogenic fibrosing alveolitis in 1984 according to the new and old rules showed a $6 \%$ increase in deaths ascribed to the disease under the new rule. Consequently the number of deaths from 1984 onwards should be scaled down by a factor of 0.94 so that the two periods are comparable. ${ }^{11}$ This fairly small change can therefore have played only a minor part in the increased number of deaths recorded.

What other factors might explain the rise in reported mortality? Changes in diagnostic practice may be a factor. For instance, some of the increase in mortality in the elderly could probably be accounted for by more detailed investigation and greater diagnostic precision in the past decade resulting in more patients being recognised as having the disease. Nevertheless, the increase also occurred in people aged 55-65, albeit to a lesser extent, and it seems unlikely that the classic pattern of the disease would have been overlooked often in this age group in the 1970s. As the disease does not have a precise definition, however, small changes in diagnostic practice, irrespective of the intensity of any investigation, might be partly responsible for changes in reported mortality. The increase in the numbers of the elderly does not account for the rise in mortality as the age standardised mortality for each sex showed the same doubling as the absolute numbers of deaths. It is thus impossible to be certain how much of the rise is a real trend and how much is an artefact due to changes in diagnostic practice or classification and coding of the disease.

Using national mortality data we looked for factors associated with death from cryptogenic fibrosing alveolitis that might have an aetiological role. Firstly, we found that deaths from the disease were commoner in men at all ages (about $2 \cdot 2: 1$ overall). The disease has been found to be predominant among men in some ${ }^{212}$ but by no means all ${ }^{13}$ surveys from specific centres. Why should this be? There are no clear genetic factors as, although familial fibrosing alveolitis has been described, ${ }^{14}$ it is uncommon clinically and no definite link with any HLA pattern has been found. ${ }^{1516}$ Increased cigarette smoking is a potential factor in the higher mortality among men and needs further study; cigarette smoking was common $(74-80 \%)$ in two studies of patients between 1950 and 1976. ${ }^{212}$ Male oriented sports and hobbies should be considered, but cryptogenic fibrosing alveolitis has numerous clinical differences from alveolitis due to extrinsic allergens. Occupational exposure to some factor, however, must be a strong contender, and support for this is provided by the regional differences found in this study, which, though small, suggest a concentration of the disease in the central industrialised areas of England and Wales. The data did not permit any further analysis of whether an urban or rural environment might be associated with mortality from the disease, though a report from Japan suggests that the disease might be commoner in rural areas. ${ }^{17}$ Likewise, the numbers were too small to permit any analysis at subregional level.

The regional differences are unlikely to be due to differences in diagnostic practice as, if anything, fibrosing lung disease may be more likely to be labelled pneumoconiosis in industrial areas, though occupational chest radiographic screening is more common in these areas. If the regional differences do indeed reflect true differences in mortality then occupational exposure must be strongly considered to be a possible aetiological factor. This might be exposure to agents previously unrecognised as being fibrogenic or occult or low exposure to known agents, such as asbestos. In this respect we found no relation between the Registrar General's social class and mortality, suggesting that those working in manual occupations were not at increased risk; residual effects of mild exposure would nevertheless be difficult to identify.

The striking increase in mortality with age raises the additional possibility that the disease is a degenerative process in the lung. If this were the case, however, why, with its characteristic clinical pattern, was it not well described before the 1940s? ${ }^{18}$ Unless it was simply unrecognised among the much more common chronic chest diseases, notably tuberculosis, a long exposure to a fairly new environmental agent is again a likely explanation. Quite possibly, however, the disease is not aetiologically homogeneous and is a stereotyped response of the lung to various undetermined factors.

This study has shown for the first time that mortality from cryptogenic fibrosing alveolitis has doubled over the past 10 years, though some of this increase could be due to changes in diagnostic practice. The findings that deaths from cryptogenic fibrosing alveolitis are commoner in men, the elderly, and the central parts of England and Wales suggest an environmental cause. Further epidemiological studies are now needed; investigations into aetiology require a more precise definition of disease and assessment of exposure to various agents.

We thank the Office of Population Censuses and Surveys for supplying data on mortality, doctors in Nottingham for their help in compiling a case register of patients with cryptogenic fibrosing alveolitis, and Dr Maurice Beaver for providing the computer records of death certificates in Nottingham.

1 Crsytal RG, Bitterman PB, Rennard SI, Hance AJ, Keogh BA. Interstitial lung diseases of unknown cause. $N$ Engl F Med 1984;310:154-66.

2 Turner-Warwick M, Burrows B, Johnson A. Cryptogenic fibrosing alveolitis response to corticosteroid treatment and its effect on survival. Thora 1980;35:593-9. 
3 Tukiainen P, Taskinen E, Holsti P, Korhola O, Valle M. Prognosis of cryptogenic fibrosing alveolitis. Thorax 1983;38:349-55.

4 Weissler JC. Idiopathic pulmonary fibrosis: cellular and molecular pathogenesis. Am F Med Sci 1989;297:91-104.

5 World Health Organisation. International classification of diseases 1975 9 th revision. Geneva: WHO, 1977.

6 Office of Population Censuses and Surveys. Mortality statistics 1979-1987. London: HMSO, 1980-8. (Series DH2, Nos 6-14.)

7 SPSS Incorporated. Statistical package for the social sciences- $X$ users guide. $2 \mathrm{nd}$ ed. New York: McGraw Hill, 1986.

8 Numerical Algorithms Group. Generalised linear interactive modelling system, release 3.77. Oxford: Royal Statistical Society, 1986

9 Office of Population Censuses and Surveys. Census 1981. National report. Great Britain part 2. London: HMSO, 1983. (CEN 81 NR(2).)

10 Johnston IDA, Bleasdale C, Hind CRK, Woodcock AA. Cryptogenic fibrosing alveolitis: accuracy of diagnostic coding in hospital admissions. Thorax 1989;44:871P.

11 Office of Population Censuses and Surveys. Mortality statistics 1984. London: HMSO, 1985. (Series DH2, No 11 .)
12 Carrington CB, Gaensler EA, Coutu RE, Fitzgerald MX, Gupta RG. Natural history and treated course of usual and desquamative interstitial pneumonia. N Engl F Med 1978;298:801-9.

13 Stack BHR, Choo-Kang YFJ, Heard BE. The prognosis of fibrosing alveolitis. Thorax 1972;27:535-42.

作

15 Fulmer JD, Sposovska MS, Von Gal ER, Crystal RG, Mittal KK. Distributio of HLA antigens in idiopathic pulmonary fibrosis. Am Rev Respir Dis 1978;118:141-7.

16 Turton CWG, Morris LM, Lawler SD, Turner-Warwick M. HLA in cryptogenic fibrosing alveolitis. Lancet 1978; i:507-8.

17 Iwai K, Nakajima T, Miyagi Y, et al. Epidemiological studies on idiopathic diffuse interstitial fibrosis of the lung. Nippon Kyobu Shikkan Gakkai Zasshi 1980;18:800-8.

18 Hamman L, Rich AR. Acute diffuse interstitial fibrosis of the lungs. Bulletin of the Johns Hopkins Hospital 1944;74:177-212.

(Accepted 15 August 1990)

\title{
Home treatment for acute psychiatric illness
}

\author{
C Dean, E M Gadd
}

\begin{abstract}
Objective-To determine the factors influencing the successful outcome of community treatment for severe acute psychiatric illnesses that are traditionally treated in hospital.

Design-All patients from a single electoral ward who were either admitted to hospital or treated at home over a two year period (1 October 1987 to 30 September 1989) were included in the study and their case notes audited. The second year of the study is reported.
\end{abstract} ham.

Setting-Electoral ward of Sparkbrook, Birming-

Subjects-99 Patients aged 16-65 with severe acute psychiatric illness.

Results -65 Patients were managed by home treatment alone; 34 required admission to hospital. The location of treatment was significantly (all $\mathbf{p}<0.05$ ) influenced by social characteristics of the patients (marital state, age (in men), ethnicity, and living alone) and by characteristics of the referral (occurring out of hours; assessment taking place at hospital or police station). DSM-III-R diagnosis was more weakly associated with outcome. Violence during the episode was significantly related to admission, although deliberate self harm was not.

Conclusions - Home treatment is feasible for most patients with acute psychiatric illness. A 24 hour on call assessment service increases the likelihood of success because admission is determined more strongly by social characteristics of the patient and the referral than by illness factors. Admission will still be required for some patients. A locally based mental health resource centre, a 24 hour on call service, an open referral system, and an active follow up policy increase the effectiveness of a home treatment service.

\section{Introduction}

Most serious psychiatric illnesses are chronic relapsing conditions. Admission to a psychiatric hospital for the acute relapses does little to influence the long term course of the diseases, and with the move away from institutional care it offers only very short respite to the relatives.

During the past 30 years several investigators have examined alternatives to hospital admission and the effect of such alternatives on patients and their relatives both during and after the episode of illness. Several studies examined the feasibility of treating patients in their own homes. Fenton et al were able to prevent admission in $62 \%$ of their 76 home care patients during a one year study, although $45 \%$ of those presenting for admission were excluded from the trial. ${ }^{1}$ Stein and Test reported a study in Madison, Wisconsin, where 130 patients who presented for admission to hospital were randomly allocated to either hospital or community treatment. ${ }^{2}$ In the year following treatment the community group had fewer admissions, fewer psychiatric symptoms, and better social functioning and reported more satisfaction than the group treated in hospital. This study was replicated by Hoult and Reynolds in Sydney, Australia, with broadly similar findings, ${ }^{3}$ and they also found that patients and their relatives reported more satisfaction with the community treatment. ${ }^{4}$ Pai and Kapur examined home treatment for schizophrenic patients in Bangalore, India, and showed a reduction in family burden at six month follow up for those who had been treated at home. ${ }^{5}$ A number of earlier studies reported similar findings: the group treated at home had a better outcome. - $^{6-8}$

Patients dependent on alcohol or drugs and those with organic brain syndromes have been excluded from all trials of home treatment. The older studies excluded patients without family or surrogate and those who were suicidal or homicidal. No study has shown that it is possible to avoid admission entirely. Grad and Sainsbury provided the only report of a total adult psychiatric service in Chichester, England, showing that an expansion in community facilities and increased domiciliary visiting by the psychiatric team greatly reduced the total number of admissions in comparison with the traditional service in Salisbury, which was demographically similar. ${ }^{9}$ None of the reported studies of home treatment were carried out in Great Britain and all were done 10-20 years ago, making it difficult to generalise the findings to current psychiatric practice in Britain.

We report a service in Birmingham that provides a total psychiatric service to a community by using home treatment principles. Central Birmingham psychiatric services are organised in sectors, with each general psychiatry consultant being responsible for patients under 65 in one or two electoral wards. CD is the consultant responsible for the inner city electoral ward of Sparkbrook, which has a population of 25728 (1981 census). Over half the inhabitants are from the New Commonwealth or Pakistan, $11 \%$ from the Irish Republic, and 35\% from the United Kingdom. The population is mostly social classes III manual, IV, or V; only $12 \%$ are in social class II or III non-manual of the Registrar General's classifications. Unemployment is Dr Dean. 